\title{
Regular eating, not intermittent fasting, is the best strategy for a healthy eating control
}

\author{
Riccardo Dalle Grave \\ Department of Eating and Weight Disorders, Villa Garda Hospital, Garda (VR), Italy
}

\section{Key words}

Intermittent fasting

Regular eating

Starvation

Life expectancy

Health disease

Aging

Eating disorders

Bulimia nervosa

Binge-eating disorder

\begin{abstract}
Intermitting fasting has been proposed as a dietary strategy to improve general health indicators, slow or reverse disease processes and aging. However, data supporting the beneficial health effects of intermittent fasting mainly derive from preclinical studies in animals and short-term clinical studies on weight loss and non-communicable disease risk factors, with no significant differences on these outcomes when intermittent fasting has been compared with moderate continuous energy restriction in humans. No data are available on the effect of intermitting fasting on clinically important outcomes in humans, such as the onset of cardiovascular diseases, cancer, and life expectancy. Instead, several studies found an association between delayed eating (i.e., spending many hours during the day without eating) and increased risk of developing episodes of overeating and binge eating. Moreover, the procedure of regular eating, adopted by the "enhanced" cognitive behavior therapy for eating disorders (CBT-E), results in a rapid decease in the frequency of binge-episodes in patients with bulimia nervosa and bingeeating disorders. These data indicate that regular eating, not intermitting fast, is the best choice for adopting a healthy eating control and avoiding the development of unregulated and, in some cases, disturbed eating behavior.
\end{abstract}

Intermittent fasting has been recently proposed as an eating pattern strategy to elicit, through the metabolic switch from the use of glucose as fuel source to the use of fatty acids and ketone bodies, the improvement of insulin resistance, obesity, dyslipidemia, high blood pressure and cognitive functions, the increased resistance to stress, the suppression of inflammation and the increase of life expectancy (de Cabo \& Mattson, 2019). These dietary practices in its more common forms (e.g., alternate fasting days; 5:2 intermittent fasting, i.e., fasting 2 days each week; daily time-restricted feeding) (Anton et al., 2018) has been proposed to treat obesity, diabetes, cardiovascular diseases, cancer, neurodegenerative diseases, asthma, multiple sclerosis, osteoarthri- tis, surgical tissue lesions and ischemic and to prolong life expectancy (de Cabo \& Mattson, 2019).

Data supporting the beneficial effects of intermittent fasting derives from preclinical studies on animals (e.g. rats) (Anton et al., 2018; Swindell, 2012). The few clinical published studies assessed only the short-term (weeks or months) effect of this intervention on weight loss and some non-communicable diseases risk factors. To date no data are available on the effect of intermitting fasting on clinically important outcomes, such as the onset of cardiovascular diseases, cancer, and lifer expectancy in humans. Furthermore, most studies did not compare the long-term effects of intermittent fasting with continuous energy re-

Copyright @ 2020 Riccardo Dalle Grave. This is an open-access article distributed under the terms of the Creative Commons Attribution License (CC BY). The use, distribution or reproduction in other forums is permitted, provided the original author(s) and the copyright owner(s) are credited and that the original publication in this journal is cited, in accordance with accepted academic practice. No use, distribution or reproduction is permitted which does not comply with these terms.

Riccardo Dalle Grave, MD (₫) rdalleg@gmail.com

Received: 11 January 2020; Accepted: 20 January 2020; Published online: 17 February 2020. doi:10.32044/ijedo.2020.02 
striction. Therefore, it is not known whether the observed positive effects on risk factors are the result of intermittent fasting or calorie restriction per se.

The only published clinical study with long-term outcome data (i.e., 24 months) on weight loss and cardiovascular risk factors is a one-year randomized parallel trial which randomized 332 adults with overweight or obesity to one of three groups: (1) continuous energy restriction (1000 kcal/ day for women and $1200 \mathrm{kcal} /$ day for men); (2) week-onweek-off energy restriction (alternating between the same energy restriction as the continuous group for one week and one week of habitual diet); or (3) 5:2 (500 kcal/day on modified fast days each week for women and $600 \mathrm{kcal} /$ day for men, the 2 days of energy restriction could be consecutive or non-consecutive). The study did not find significant differences in weight loss and in the modification of the cardiovascular risk factors among the three groups at both 12 and 24 months (Headland, Clifton, \& Keogh, 2019, 2020).

Other trials with shorter duration achieved the same conclusion as the Headland et al. study (2020). For example, a short-term randomized study, comparing alternate-day fasting with moderate daily caloric restriction in adults with obesity, found no significant between-group differences in change in weight body composition, lipids and insulin sensitivity index after 8-weeks of treatment, and no significant differences in weight regain after 24 weeks of unsupervised follow-up (Catenacci et al., 2016). The same conclusion was reached by a 12 -month trial that found no significant differences in adherence, weight loss and maintenance, and cardiovascular protection between intermittent fasting $(25 \%$ of energy needs on fast days; $125 \%$ of energy needs on alternating "feast days") compared to a moderate continuous energy restriction (75\% of energy needs every day) (Trepanowski et al., 2017). The results of these studies confirm the conclusions of a systematic metanalytical review of 9 studies of a minimum duration of 6 months, in which no difference in weight loss was observed between continuous calorie restriction and intermittent fasting (Headland, Clifton, Carter, \& Keogh, 2016).

Although there are no significant differences in terms of weight loss and improvement of cardiovascular risk factors between intermittent fasting and continuous energy restriction, several studies found an association between delayed eating (i.e., spending many hours during the day without eating) and increased risk of developing episodes of overeating and binge eating. A laboratory experiment found that participants deprived of food for 6-hour consumed significantly more calories at the buffet compared to subjects in the 1-hour food deprivation condition (Tel- ch \& Agras, 1996). Studies on binge-eating disorder (BED) have observed that patients with overweight and BED who ate three meals per day weighed significantly less, and had significantly fewer binge eating episodes, than those who did not regularly eat three meals per day (Masheb \& Grilo, 2006). Moreover, regular consumption of breakfast, lunch and dinner was significantly correlated with a lower body mass index in people with obesity and BED (Masheb, Grilo, \& White, 2011). Research has also shown that dieting significantly predicted future weight gain in normal weight individuals (Lowe, Doshi, Katterman, \& Feig, 2013), and was a risk factor for the development of eating disorders of clinical severity (Schaumberg \& Anderson, 2016; Stice, 2016).

The most important evidence in favor of regular eating pattern $(3+2+0)$, however, derives from the results of studies that assessed the efficacy and effectiveness of "enhanced" cognitive behavioral therapy (CBT-E) for eating disorders (Dalle Grave \& Calugi, 2020; Fairburn, 2008). CBT-E is based on a theory postulating that delayed eating and the adoption of extreme and rigid dietary rules are the most potent mechanisms maintaining binge-eating episodes (Fairburn, Cooper, \& Shafran, 2003). For this reason, a key procedure adopted by CBT-E is "regular eating" that suggest patients to plan in advance 3 main meals (breakfast, lunch and dinner) and 2 snacks (mid-morning snack and mid-afternoon snack) and not eating between these intervals: a procedure also called $3+2+0$. Randomized and cohort studies have shown that regular eating procedures result in a rapid decrease in the frequency of binge-episodes in patients with bulimia nervosa (Dalle Grave, Calugi, Sartirana, \& Fairburn, 2015; Fairburn et al., 2009) and BED (Grilo \& Masheb, 2005). A study assessing the efficacy of CBT for bulimia nervosa has also found that participants who ate 80 meals and at least 21 afternoon snacks in the previous month reported the highest abstinence rate (70\%) from binge-eating episodes (Shah, Passi, Bryson, \& Agras, 2005). This finding was also observed in a study using CBT guided self-help in patients with binge-eating episodes (Zendegui, West, \& Zandberg, 2014). These results led to affirm that regular eating is probably the single most important procedure in the treatment of eating disorders with binge-eating episodes (Fairburn, 2013).

In conclusion, there are no evidences, supported by long-term randomized controlled trials, that intermittent fasting produces additional clinical benefits, compared to healthy and regular eating patterns, and greater weight loss, compared to a moderate continuous energy restriction. Instead, there are some evidences that delayed eating increases the risk of developing episodes of overeating 
and binge eating. For these reasons, it is inappropriate to recommend intermitting fasting as a healthy eating practice. Regular eating, preferably based on the Mediterranean diet model (Estruch et al., 2018), is the best choice to reduce the risk of disease and to avoid the development of unregulated and, in some cases, disturbed eating behavior.

\section{References}

Anton, S. D., Moehl, K., Donahoo, W. T., Marosi, K., Lee, S. A., Mainous, A. G., 3rd, ... Mattson, M. P. (2018). Flipping the Metabolic Switch: Understanding and Applying the Health Benefits of Fasting. Obesity (Silver Spring), 26(2), 254-268. doi:10.1002/oby.22065

Catenacci, V. A., Pan, Z., Ostendorf, D., Brannon, S., Gozansky, W. S., Mattson, M. P., . . . Troy Donahoo, W. (2016). A randomized pilot study comparing zero-calorie alternateday fasting to daily caloric restriction in adults with obesity. Obesity (Silver Spring), 24(9), 1874-1883. doi:10.1002/ oby. 21581

Dalle Grave, R., \& Calugi, S. (2020). Cognitive behavior therapy for adolescents with eating disorders. New York: Guilford Press.

Dalle Grave, R., Calugi, S., Sartirana, M., \& Fairburn, C. G. (2015). Transdiagnostic cognitive behaviour therapy for adolescents with an eating disorder who are not underweight. Behaviour Research and Therapy, 73, 79-82. doi:10.1016/j.brat.2015.07.014

de Cabo, R., \& Mattson, M. P. (2019). Effects of intermittent fasting on health, aging, and disease. New England Journal of Medicine, 381(26), 2541-2551. doi:10.1056/NEJMra1905136

Estruch, R., Ros, E., Salas-Salvado, J., Covas, M. I., Corella, D., Aros, F., . . . Investigators, P. S. (2018). Primary prevention of cardiovascular disease with a mediterranean diet supplemented with extra-virgin olive oil or nut. New England Journal of Medicine, 378(25), e34. doi:10.1056/ NEJMoa1800389

Fairburn, C. G. (2008). Cognitive behavior therapy and eating disorders. New York: Guilford Press.

Fairburn, C. G. (2013). Overcoming binge eating, Second Edition. New York: Guilford Press.

Fairburn, C. G., Cooper, Z., Doll, H. A., O’Connor, M. E., Bohn, K., Hawker, D. M., .. . Palmer, R. L. (2009). Transdiagnostic cognitive-behavioral therapy for patients with eating disorders: a two-site trial with 60-week follow-up. American Journal of Psychiatry, 166(3), 311-319. doi:10.1176/appi. ajp.2008.08040608

Fairburn, C. G., Cooper, Z., \& Shafran, R. (2003). Cognitive behaviour therapy for eating disorders: a "transdiagnostic" theory and treatment. Behaviour Research and Therapy, 41(5), 509-528.

Grilo, C. M., \& Masheb, R. M. (2005). A randomized controlled comparison of guided self-help cognitive behavioral therapy and behavioral weight loss for binge eating disorder. Behaviour Research and Therapy, 43(11), 1509-1525. doi:10.1016/j.brat.2004.11.010
Headland, M., Clifton, P. M., Carter, S., \& Keogh, J. B. (2016). Weight-Loss Outcomes: A Systematic Review and MetaAnalysis of Intermittent Energy Restriction Trials Lasting a Minimum of 6 Months. Nutrients, 8(6). doi:10.3390/ nu8060354

Headland, M. L., Clifton, P. M., \& Keogh, J. B. (2019). Effect of intermittent compared to continuous energy restriction on weight loss and weight maintenance after 12 months in healthy overweight or obese adults. International Journal of Obesity, 43(10), 2028-2036. doi:10.1038/s41366-018-0247-2

Headland, M. L., Clifton, P. M., \& Keogh, J. B. (2020). Impact of intermittent vs. continuous energy restriction on weight and cardiometabolic factors: a 12-month follow-up. International Journal of Obesity doi:10.1038/s41366-0200525-7

Lowe, M. R., Doshi, S. D., Katterman, S. N., \& Feig, E. H. (2013). Dieting and restrained eating as prospective predictors of weight gain. Frontiers in Psychology, 4, 577. doi:10.3389/ fpsyg.2013.00577

Masheb, R. M., \& Grilo, C. M. (2006). Eating patterns and breakfast consumption in obese patients with binge eating disorder. Behaviour Research and Therapy, 44(11), 15451553. doi:10.1016/j.brat.2005.10.013

Masheb, R. M., Grilo, C. M., \& White, M. A. (2011). An examination of eating patterns in community women with bulimia nervosa and binge eating disorder. International Journal of Eating Disorders, 44(7), 618-624. doi:10.1002/ eat.20853

Schaumberg, K., \& Anderson, D. (2016). Dietary restraint and weight loss as risk factors for eating pathology. Eating Behaviors, 23, 97-103. doi:10.1016/j.eatbeh.2016.08.009

Shah, N., Passi, V., Bryson, S., \& Agras, W. S. (2005). Patterns of eating and abstinence in women treated for bulimia nervosa. International Journal of Eating Disorders, 38(4), 330-334. doi:10.1002/eat.20204

Stice, E. (2016). Interactive and Mediational Etiologic Models of Eating Disorder Onset: Evidence from Prospective Studies. Annual Review of Clinical Psychology, 12, 359-381. doi:10.1146/ annurev-clinpsy-021815-093317

Swindell, W. R. (2012). Dietary restriction in rats and mice: a meta-analysis and review of the evidence for genotypedependent effects on lifespan. Ageing Research Reviews, 11(2), 254-270. doi:10.1016/j.arr.2011.12.006

Telch, C. F., \& Agras, W. S. (1996). The effects of short-term food deprivation on caloric intake in eating-disordered subjects. Appetite, 26(3), 221-233. doi:10.1006/appe.1996.0017

Trepanowski, J. F., Kroeger, C. M., Barnosky, A., Klempel, M. C., Bhutani, S., Hoddy, K. K., . . Varady, K. A. (2017). Effect of alternate-day fasting on weight loss, weight maintenance, and cardioprotection among metabolically healthy obese adults: A randomized clinical trial. JAMA Internal Medicine, 177(7), 930-938. doi:10.1001/jamainternmed.2017.0936

Zendegui, E. A., West, J. A., \& Zandberg, L. J. (2014). Binge eating frequency and regular eating adherence: the role of eating pattern in cognitive behavioral guided self-help. Eating Behaviors, 15(2), 241-243. doi:10.1016/j.eatbeh.2014.03.002 